\title{
A NEW NUMERICAL APPROACH OF A LUBRICATION FREE BOUNDARY PROBLEM
}

\author{
Sixto J. Alvarez \& Rachid Oujja ${ }^{1}$ \\ Departmento de matemática Aplicada, Universidad Complutense \\ Facultad de Ciencias Matemáticas \\ 28040 Madrid Spain
}

Abstract. A combination of a semidiscretized scheme and an iterative method is applied to a free boundary problem. The considered problem is issued from a cavitation model in lubrication theory and treats on the determination of the cavitation area. Estimates $L^{\infty}(\Omega)$ which provide the convergence of the approximated solution is obtained. Finally, some numerical test examples are presented to illustrate the good performance of the method.

keywords: Cavitation theory, Free boundary problem, Numerical solution.

\section{INTRODUCTION AND MATHEMATICAL MODEL}

In the present paper, we apply a new numerical method to solve a nonlinear free boundary problem related to the cavitation phenomenon in a journal bearing. The approximate solution is obtained through a process of successive approximations, where at each step a system of differential equations is solved. The one-dimensional case, which corresponds to the particular case of an infinite journal bearing, is treated in [2] where an analysis of the iterative method and some graphical results are given. In this paper we consider the general case where a twodimensional problem must be solved.

To describe the cavitation phenomenon two unknowns are required: The pressure, $p$, in a thin film of lubricant contained in the narrow gap between the surface of the bearing and the surface of the journal in relative motion, and also the fraction of volume occupied by the lubricant, $\theta$. The geometry of the bearing implies that $p$ and $\theta$ are $2 \pi-$ periodic in the circumferential

\footnotetext{
${ }^{1}$ Supported by the postdoctoral Fellowship of Comunidad de Madrid Spain

${ }^{1}$ E-mail address: Rachid_Oujja@mat.ucm.es
} 
coordinate $x$. The film thickness is denoted by

$$
h(x)=1+\alpha \cos x \text { with } \alpha \text { a constant in }(0,1)
$$

We consider here the Elrod-Adams model [5] which is considered the most realistic; and leads to the following strong formulation:

Let $\Omega$ be the domain $(0,2 \pi) \times(0,1)$ which represents the internal bearing surface, $\Gamma$ its boundary, let $\Gamma_{0}$ and $\Gamma_{1}$ be defined by

$$
\Gamma_{0}=(0,2 \pi) \times\{0\}, \quad \Gamma_{1}=(0,2 \pi) \times\{1\}
$$

and let $\Omega_{0}$ be the region of cavitation (see Fig. 1).

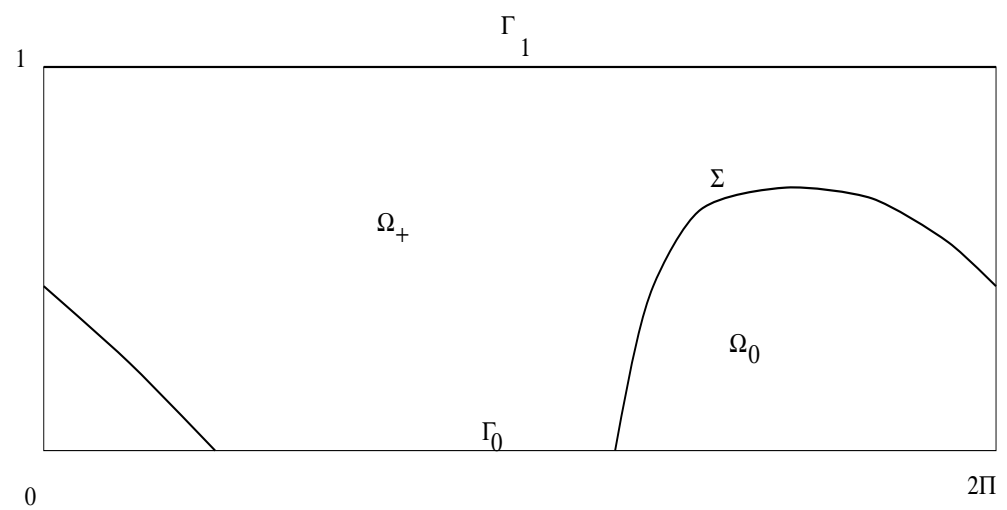

Figure 1: The domain $\Omega$

The problem is to find two functions, $p$ and $\theta$, defined in $\Omega$ such that

$$
\begin{aligned}
& \frac{\partial}{\partial x}\left(h^{3} \frac{\partial p}{\partial x}\right)+\frac{\partial}{\partial y}\left(h^{3} \frac{\partial p}{\partial y}\right)=\frac{d h}{d x} \quad p>0, \quad \theta=1 \text { in } \Omega \backslash \Omega_{0} \\
& \frac{\partial(h \theta)}{\partial x}=0 \quad p=0, \quad 0 \leq \theta \leq 1 \text { in } \Omega_{0} \\
& h^{3} \frac{\partial p}{\partial n}=(1-\theta) h \cos (n, x) p=0, \text { on } \Sigma=\partial \Omega_{0} \cap \Omega \\
& p=0 \quad \text { on } \quad \Gamma_{0}, \quad p=p_{a}>0 \quad \text { on } \Gamma_{1}
\end{aligned}
$$

Where $n$ is the outward normal On $\Sigma$.

Let us introduce the sets

$$
\begin{gathered}
V_{0}=\left\{\xi \in H^{1}(\Omega),\left.\xi\right|_{\Gamma_{0} \cup \Gamma_{1}}=0, \xi \quad \text { is } 2 \pi x-\text { periodic }\right\} \\
V_{a}=\left\{\xi \in H^{1}(\Omega),\left.\xi\right|_{\Gamma_{0}}=0,\left.\xi\right|_{\Gamma_{1}}=p_{a}, \xi \quad \text { is } 2 \pi x-\text { periodic }\right\}
\end{gathered}
$$


The weak formulation of the problem can be deduced ( see [1]) as follows:

Find a pair of functions $(p, \gamma)$ such that:

$$
\begin{aligned}
& (p, \gamma) \in V_{a} \times L^{\infty}(\Omega) \\
& p \geq 0 \quad \text { and } \quad H(p) \leq \gamma \leq 1 \text { a.e in } \Omega \\
& \int_{\Omega} h^{3} \nabla p \nabla \xi=\int_{\Omega} h \gamma \xi_{x}, \text { for each } \xi \in V_{0},
\end{aligned}
$$

where $H$ is the Heaviside graph. Existence and uniqueness of a weak solution of this problem is well known, and it has been treated in many papers ([1], [3]).

\section{Discretization By A Method of Lines}

Let $q \in V_{0}$ be a function satisfying the variational equation:

$$
\int_{\Omega} h^{3} \nabla q \nabla \xi=\int_{\Omega} h \xi_{x}, \quad \forall \quad \xi \in V_{0}
$$

It is well known from classical results on variational inequalities that the solution $q$ exists and is unique. And we have (see [7]) that

$$
q \in C^{1, \alpha}(\Omega), \text { For some } \alpha>0
$$

being $C^{1, \alpha}(\Omega)$ the space of functions on $\Omega$ with uniformly Holder continuous first order derivatives.

By other hand, we have

Lemma 2.1. The solution $q$ satisfies $\int_{0}^{2 \pi} h^{3}(x) q(x, y) d x=0$ for all $y \in(0,1)$.

Proof. For a test function $\xi=\xi(y) \in \mathcal{D}(0,1)$ in $(2.1)$ we have

$$
\int_{\Omega} h^{3} \frac{\partial q}{\partial y} \xi^{\prime}=0 .
$$

Now by integrating by parts, and separating the integration variables we obtain

$$
\int_{0}^{1}\left(\int_{0}^{2 \pi} h^{3} q\right) \xi^{\prime \prime}=0
$$

and we deduce that $\int_{0}^{2 \pi} h^{3}(x) q(x, y) d x=A y+B$.

Since $\int_{0}^{2 \pi} h^{3}(x) q(x, 0) d x=\int_{0}^{2 \pi} h^{3}(x) q(x, 1) d x=0$ we obtain that $A=B=0$.

We will show now, that a method of lines can be used to build a surface which can be related to the solution $q$ of the variational equation (2.1). 
Let $\Delta y=\frac{1}{N+1}, N>0$, and $V_{N}$ the subspace of $V_{0}$ of functions on $\Omega$ which are linear in the variable $y$ over each interval $\left(y_{i}, y_{i+1}\right)$, where $y_{i}=i \Delta y$ for $i=0,1, \ldots, N+1$ :

$$
V_{N}=\left\{\xi_{n} \in V_{0} \text { such that }\left.\xi_{n}\right|_{\left(y_{i}, y_{i+1}\right)} \in P_{1}\left(\left[y_{i}, y_{i+1}\right]\right), i=0, \ldots, N\right\}
$$

and let us consider the approximated problem:

$$
\int_{\Omega} h^{3} \nabla q_{N} \nabla \xi_{n}=\int_{\Omega} h \frac{\partial \xi_{n}}{\partial x}, \quad \forall \quad \xi_{n} \in V_{N}
$$

Assume that $\left\{\phi_{j}, j=1, \ldots, N\right\}$ is a basis of $V_{N}$ and $\phi_{j}\left(y_{i}\right)=\delta_{i, j}$ for $1 \leq i, j \leq N$. For all function $p_{N} \in V_{N}$ we have

$$
p_{N}(x, y)=\sum_{i=0}^{N+1} p_{N}\left(x, y_{i}\right) \phi_{i}(y) .
$$

Theorem 1. The solution $q_{N}$ of the approximated problem (2.3) converges to the solution $q$ of the continue problem (2.1).

Proof. In order to establish convergence, classical results may be applied (see for example [6]). We observe that the bilinear form

$$
B(u, v)=\int_{\Omega} h^{3} \nabla u \nabla v
$$

is bounded and coercive in $V_{0}$. Hence there exists positive constants $c$ and $k$ such that:

$$
c\|u\|^{2} \leq B(u, u) ; \quad|B(u, v)| \leq K\|u\|\|v\|,
$$

Where $\|$.$\| is the usual norm on H^{1}(\Omega)$. If we define the operator $A$ from $V_{0}$ into $H^{-1}(\Omega)$ by

$$
B(u, v)=(A u, v) \text { for each } v \in V_{0}
$$

it follows from (2.1) and (2.3) that:

$$
\begin{array}{r}
B(q, \xi)=\left(-h^{\prime}, \xi\right) \quad \forall \quad \xi \in V_{0} \\
B\left(q_{N}, \xi_{n}\right)=\left(-h^{\prime}, \xi_{n}\right) \quad \forall \quad \xi_{n} \in V_{N} .
\end{array}
$$

As in [6] we can obtain

$$
\left\|q-q_{N}\right\|^{2} \leq \frac{K^{2}}{c^{2}}\left\|q-v_{n}\right\|^{2}+\frac{2}{c}\left\|h^{\prime}+A q\right\|_{0}\left(\left\|q-v_{n}\right\|_{0}+\left\|q_{N}-v\right\|_{0}\right) .
$$

where $\|\cdot\|_{0}$ denotes the $L_{2}$ norm on $\Omega$. Here $v_{n}$ and $v$ are arbitrary in $V_{N}$ and $V_{0}$, respectively. If in particular $v_{n}$ is the function which agrees with $q$ on the lines $y=y_{i}, i=0, \ldots, N+1$, and from the regularity of the function $q$ it follows immediately that:

$$
\left\|q-v_{n}\right\| \leq C \Delta y, \quad\left\|q-v_{n}\right\|_{0} \leq C \Delta y^{2}
$$


where $C$ depends on the $H^{2}(\Omega)$ norm of $q$. We also may conclude from the smoothness of $q$ that $\left\|h^{\prime}+A q\right\|_{0} \leq C$.

Since $V_{N} \subset V_{0}$ we may set $v=q_{N}$ and simplify the above inequality to $\left\|q-q_{N}\right\| \leq C \Delta y$ for some constant $C$. Hence, we get the convergence of $q_{N}$ to the solution $q$ in $H^{1}(\Omega)$.

Now, by a straightforward calculation, applying summation by parts and some algebraic rearrangement we show that

$$
\begin{aligned}
& \int_{\Omega} h^{3} \nabla q_{N} \nabla \xi_{n}=\int_{0}^{2 \pi} h^{3} \Delta y \sum_{i=1}^{i=N+1} \frac{q_{i}-q_{i-1}}{\Delta y} \frac{\xi_{i}-\xi_{i-1}}{\Delta y} \\
& +\int_{0}^{2 \pi} h^{3} \sum_{i=1}^{i=N+1} \int_{y_{i-1}}^{y_{i}}\left(q_{i}^{\prime} \phi_{i}+q_{i-1}^{\prime} \phi_{i-1}\right)\left(\xi_{i}^{\prime} \phi_{i}+\xi_{i-1}^{\prime} \phi_{i-1}\right) \\
& =\int_{0}^{2 \pi} h^{3} \Delta y \sum_{i=1}^{i=N} \frac{-q_{i-1}+2 q_{i}-q_{i+1}}{\Delta y^{2}} \xi_{i} \\
& +\int_{0}^{2 \pi} h^{3} \Delta y \sum_{i=1}^{i=N+1}\left(\frac{1}{3} q_{i}^{\prime} \xi_{i}^{\prime}+\frac{1}{3} q_{i-1}^{\prime} \xi_{i-1}^{\prime}+\frac{1}{6} q_{i}^{\prime} \xi_{i-1}^{\prime}+\frac{1}{6} q_{i-1}^{\prime} \xi_{i}^{\prime}\right) \\
& =\int_{0}^{2 \pi} h^{3} \Delta y \sum_{i=1}^{i=N} \frac{-q_{i-1}+2 q_{i}-q_{i+1}}{\Delta y^{2}} \xi_{i} \\
& +\int_{0}^{2 \pi} h^{3} \Delta y \sum_{i=1}^{i=N+1}\left(\frac{1}{3} q_{i}^{\prime} \xi_{i}^{\prime}+\frac{1}{3} q_{i-1}^{\prime} \xi_{i-1}^{\prime}+\frac{1}{6} q_{i}^{\prime} \xi_{i}^{\prime}+\frac{1}{6} q_{i-1}^{\prime} \xi_{i-1}^{\prime}\right) \\
& +\int_{0}^{2 \pi} h^{3} \Delta y \sum_{i=1}^{i=N+1}\left(\frac{1}{6} q_{i}^{\prime}\left(\xi_{i-1}^{\prime}-\xi_{i}^{\prime}\right)+\frac{1}{6} q_{i-1}^{\prime}\left(\xi_{i}^{\prime}-\xi_{i-1}^{\prime}\right)\right) \\
& =\sum_{i=1}^{i=N} \Delta y\left[\int_{0}^{2 \pi} h^{3} \frac{-q_{i-1}+2 q_{i}-q_{i+1}}{\Delta y^{2}} \xi_{i}+\int_{0}^{2 \pi} h^{3} q_{i}^{\prime} \xi_{i}^{\prime}\right] \\
& -\sum_{i=1}^{i=N+1} \Delta y \int_{0}^{2 \pi} h^{3}\left(q_{i}^{\prime}-q_{i-1}^{\prime}\right)\left(\xi_{i}^{\prime}-\xi_{i-1}^{\prime}\right)
\end{aligned}
$$


where $\xi_{i}=\xi_{n}\left(x, y_{i}\right)$. Recall that $q_{0}=q_{N+1}=\xi_{0}=\xi_{N+1}=0$.

For the right hand side we have

$$
\begin{aligned}
\int_{\Omega} h \frac{\partial \xi_{n}}{\partial x} & =\int_{0}^{2 \pi} h \sum_{i=1}^{i=N+1} \int_{y_{i-1}}^{y_{i}}\left(\xi_{i}^{\prime} \phi_{i}+\xi_{i-1}^{\prime} \phi_{i-1}\right) \\
& =\int_{0}^{2 \pi} h \frac{\Delta y}{2} \sum_{i=1}^{i=N+1}\left(\xi_{i}^{\prime}+\xi_{i-1}^{\prime}\right) \\
& =\int_{0}^{2 \pi} h \Delta y \sum_{i=1}^{i=N} \xi_{i}^{\prime} \\
& =\sum_{i=1}^{i=N} \Delta y \int_{0}^{2 \pi} h \xi_{i}^{\prime}
\end{aligned}
$$

Thus, equation (2.3) is equivalent to:

$$
\sum_{i=1}^{N} \Delta y\left[\int_{0}^{2 \pi} h^{3} \frac{-q_{i-1}+2 q_{i}-q_{i+1}}{\Delta y^{2}} \xi_{i}+\int_{0}^{2 \pi} h^{3} q_{i}^{\prime} \xi_{i}^{\prime}\right]=\sum_{i=1}^{N} \Delta y \int_{0}^{2 \pi} h \xi_{i}^{\prime}+R_{N}
$$

where

$$
R_{N}=\sum_{i=1}^{N+1} \Delta y \int_{0}^{2 \pi} h^{3}\left(q_{i}^{\prime}-q_{i-1}^{\prime}\right)\left(\xi_{i}^{\prime}-\xi_{i-1}^{\prime}\right) .
$$

and $q_{i}$ an approximation of $q\left(x, y_{i}\right)$. Now, from $(2.2)$ we have $\left|q_{i}^{\prime}-q_{i-1}^{\prime}\right| \leq \Delta y^{\alpha}$ for some $\alpha>0$, then we get easily

$$
\lim _{N \rightarrow \infty} R_{N}=0
$$

Finally, equation (2.3) can be reduced to solve the following system of coupled one dimensional variational equations:

$$
\begin{aligned}
& \int_{0}^{2 \pi} h^{3} q_{i}^{\prime} \xi^{\prime}+\frac{2}{\Delta y^{2}} \int_{0}^{2 \pi} h^{3} q_{i} \xi=\int_{0}^{2 \pi} h \xi^{\prime}+\frac{1}{\Delta y^{2}} \int_{0}^{2 \pi} h^{3}\left[q_{i+1}+q_{i-1}\right] \xi \quad i=1, \ldots, N \\
& q_{0}(x)=0, \quad q_{N+1}(x)=0
\end{aligned}
$$

where the test functions $\xi$ now belong to $H^{1}(0,2 \pi)$ and they are $2 \pi$ periodic. Taking into account lemma (2.1), each function $q_{i}$ satisfies

$$
\int_{0}^{2 \pi} h^{3} q_{i}=0
$$


Remark 2.1. Note that to approximate $q+p_{a} y$, the conditions (2.7) and (2.8) must be substituted respectively by

$$
\begin{aligned}
& q_{0}(x)=0 \quad q_{N+1}(x)=p_{a} \\
& \int_{0}^{2 \pi} h^{3} q_{i}=p_{a} i \Delta y \int_{0}^{2 \pi} h^{3}
\end{aligned}
$$

\section{ITERATIVE ALGORITHM}

To solve system (2.6) with the boundary condition (2.9), we can employ a Gauss-Seidel iterative algorithm. Thus, for $\mathrm{i}=1, \ldots, \mathrm{N}$ and $\mathrm{k}=1,2, \ldots$ we shall solve

$$
\begin{aligned}
& \int_{0}^{2 \pi} h^{3} q_{i}^{k^{\prime}} \xi^{\prime}+\frac{2}{\Delta y^{2}} \int_{0}^{2 \pi} h^{3} q_{i}^{k} \xi=\int_{0}^{2 \pi} h \xi^{\prime}+\frac{1}{\Delta y^{2}} \int_{0}^{2 \pi} h^{3}\left[q_{i+1}^{k-1}+q_{i-1}^{k}\right] \xi \\
& q_{0}^{k}(x)=0, \quad q_{N+1}^{k}(x)=p_{a} \quad \forall k
\end{aligned}
$$

where $\xi \in H^{1}(0,2 \pi)$ and is $2 \pi$ periodic. Taking into account condition (2.10) each solution $q_{i}^{k}$ belongs to the set

$$
W_{i}=\left\{v_{i} \in H^{1}(0,2 \pi), v_{i} \text { is } 2 \pi-x \text { periodic and } \int_{0}^{2 \pi} h^{3} v_{i}=p_{a} i \Delta y \int_{0}^{2 \pi} h^{3}\right\} .
$$

Thus, to start the algorithm we take

$$
q_{i}^{0}(x)=p_{a} i \Delta y \quad i=0,1, \ldots, N+1
$$

and at each step we solve (3.1)-(3.2).

In Fig. 2 we give the result obtained after $k=40$ iterations.

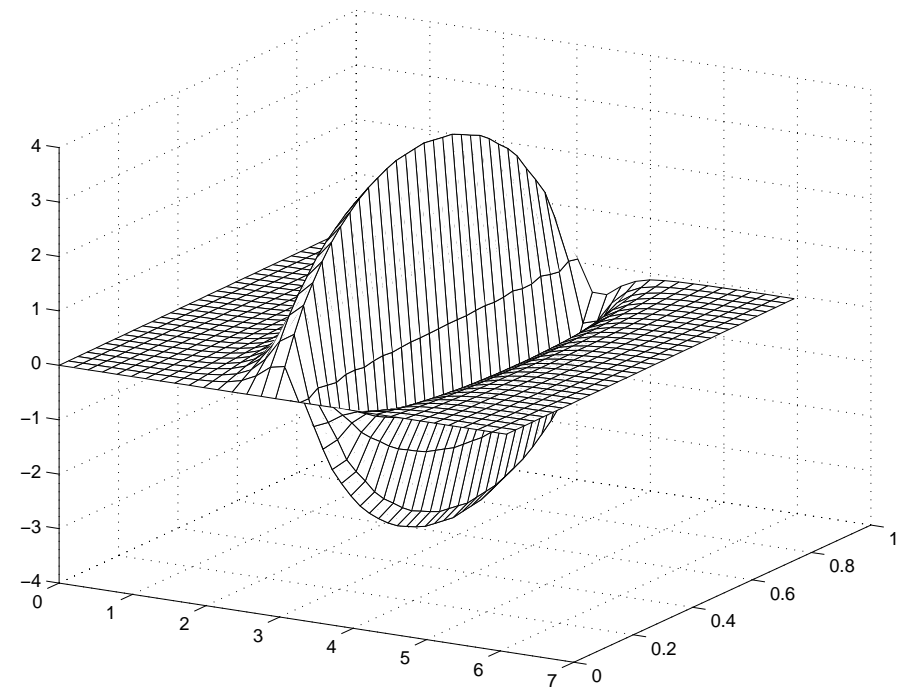

Fig. 2: Function $q$ 
3.1. A modified iterative Gauss-Seidel algorithm. Now, we take $q_{i}^{0}(x)=q\left(x, y_{i}\right)$,

$i=1, \ldots, N$ being $q$ the function of Fig. 2 ; and we apply a variation of the Gauss-Seidel iterative algorithm applied in (3.1)-(3.2). Thus, for $\mathrm{i}=1, \ldots, \mathrm{N}$ and $\mathrm{k}=1,2, \ldots$ we shall solve

$$
\begin{aligned}
& \int_{0}^{2 \pi} h^{3} q_{i}^{k^{\prime}} \xi^{\prime}+\frac{2}{\Delta y^{2}} \int_{0}^{2 \pi} h^{3} q_{i}^{k} \xi=\int_{0}^{2 \pi} h_{k-1} \xi^{\prime}+\frac{1}{\Delta y^{2}} \int_{0}^{2 \pi} h^{3}\left[q_{i+1}^{k-1}+q_{i-1}^{k}\right] \xi \\
& q_{0}^{k}(x)=0, \quad q_{N+1}^{k}(x)=p_{a} \quad \forall k
\end{aligned}
$$

being

$$
h_{k-1}(x)=h(x)+\left(h\left(a_{k-1}-h(x)\right) \chi\left(\left[a_{k-1}, b_{k-1}\right]\right),\right.
$$

where $a_{k-1} \in(\pi, 2 \pi)$ and $b_{k-1}>a_{k-1}$ (modulus $\left.2 \pi\right)$ satisfying:

$$
\left(q_{i}^{k-1}\right)^{\prime}\left(a_{k-1}\right)=0 \quad \text { and } \quad q_{i}^{k-1}\left(b_{k-1}\right)=0
$$

and $q_{i}^{k-1}$ is an increasing function in the interval $\left(a_{k-1}, b_{k-1}\right) \cdot \chi\left(\left[a_{k-1}, b_{k-1}\right]\right)$ is the characteristic function of the interval $\left[a_{k-1}, b_{k-1}\right]$.

3.2. Weak convergence of the solutions. It will be convenient to renorm $W_{i}$ with

$$
\|\xi\|_{i}=[<\xi, \xi>+(\xi, \xi)]^{1 / 2}
$$

where

$$
<\xi, \psi>=(1-\alpha)^{3} \int_{0}^{2 \pi} \xi^{\prime} \psi^{\prime} d x
$$

and

$$
(\xi, \psi)=\frac{2}{\Delta y^{2}} \int_{0}^{2 \pi} h^{3} \xi \psi d x
$$

And let us introduce the Banach space $W=W_{1} \times \ldots \times W_{N}$ with elements

$U=\left(U_{1}, \ldots, U_{N}\right), U_{i} \in W_{i}$ and norm

$$
\|U\|=\max _{1 \leq i \leq N}\left\|U_{i}\right\|_{i}
$$

Proposition 3.1. The sequence $\left(q^{k}\right)_{k \geq 0}$ is bounded in the space $W$.

Proof. It is straightforward to establish that for given $q_{i-1}^{k}$ and $q_{i+1}^{k-1}$, problem (3.3)-(3.4) has a unique weak solution $q_{i}^{k} \in H^{1}(0,2 \pi)$.

Without lose of generality, we will suppose that

$$
q_{i}^{k}\left(x_{0}\right)=0 \text { for some } x_{0} \in(0,2 \pi) .
$$


Otherwise, if $m=\max _{1 \leq i \leq N} \min _{[0,2 \pi])}\left(q_{i}^{k}\right)$, we can consider the function $q_{i}^{k}-m$ which solve the equation:

$\int_{0}^{2 \pi} h^{3}\left(q_{i}^{k}-m\right)^{\prime} \xi^{\prime}+\frac{2}{\Delta y^{2}} \int_{0}^{2 \pi} h^{3}\left(q_{i}^{k}-m\right) \xi=\int_{0}^{2 \pi} h_{k-1} \xi^{\prime}+\frac{1}{\Delta y^{2}} \int_{0}^{2 \pi} h^{3}\left[\left(q_{i+1}^{k-1}-m\right)+\left(q_{i-1}^{k}-m\right)\right] \xi$

If we take $\xi=q_{i}^{k}$ in (3.3) we get:

$$
\int_{0}^{2 \pi} h^{3} q_{i}^{k^{\prime}} q_{i}^{k^{\prime}}+\frac{2}{\Delta y^{2}} \int_{0}^{2 \pi} h^{3} q_{i}^{k} q_{i}^{k}=\int_{0}^{2 \pi} h_{k-1} q_{i}^{k^{\prime}}+\frac{1}{\Delta y^{2}} \int_{0}^{2 \pi} h^{3}\left[q_{i+1}^{k-1}+q_{i-1}^{k}\right] q_{i}^{k}
$$

That implies by applying the Holder inequality:

$$
\begin{aligned}
\left\|q_{i}^{k}\right\|_{i}^{2} & \leq \int_{0}^{2 \pi} h^{3} q_{i}^{k^{\prime}} q_{i}^{k^{\prime}}+\frac{2}{\Delta y^{2}} \int_{0}^{2 \pi} h^{3} q_{i}^{k} q_{i}^{k} \\
& =\int_{0}^{2 \pi} h_{k-1}^{\prime} q_{i}^{k}+\frac{1}{\Delta y^{2}} \int_{0}^{2 \pi} h^{3}\left[q_{i+1}^{k-1}+q_{i-1}^{k}\right] q_{i}^{k} \\
& =-\frac{\Delta y^{2}}{2}\left(\frac{h_{k-1}^{\prime}}{h^{3}}, q_{i}^{k}\right)+\frac{1}{2}\left(q_{i+1}^{k-1}, q_{i}^{k}\right)+\frac{1}{2}\left(q_{i-1}^{k}, q_{i}^{k}\right) \\
& \leq \frac{\Delta y^{2}}{2}\left(\frac{h_{k-1}^{\prime}}{h^{3}}, \frac{h_{k-1}^{\prime}}{h^{3}}\right)^{1 / 2}\left(q_{i}^{k}, q_{i}^{k}\right)^{1 / 2}+\frac{1}{2}\left(q_{i+1}^{k-1}, q_{i+1}^{k-1}\right)^{1 / 2}\left(q_{i}^{k}, q_{i}^{k}\right)^{1 / 2}+\frac{1}{2}\left(q_{i-1}^{k}, q_{i-1}^{k}\right)^{1 / 2}\left(q_{i}^{k}, q_{i}^{k}\right)^{1 / 2}
\end{aligned}
$$

By other hand, from consideration (3.5) we can use the Poincaré inequality and get:

$$
\begin{aligned}
\left(q_{i}^{k}, q_{i}^{k}\right) & =\frac{2}{\Delta y^{2}} \int_{0}^{2 \pi} h^{3} q_{i}^{k^{2}} \\
& \leq \frac{2(1+\alpha)^{3}}{\Delta y^{2}} \int_{0}^{2 \pi} q_{i}^{k^{2}} \\
& \leq \frac{2(1+\alpha)^{3}}{\Delta y^{2}} 2 \pi^{2} \int_{0}^{2 \pi} q_{i}^{k^{\prime 2}} \\
& =\frac{4 \pi^{2}(1+\alpha)^{3}}{\Delta y^{2}(1-\alpha)^{3}}\left\langle q_{i}^{k}, q_{i}^{k}\right\rangle
\end{aligned}
$$

whence

$$
\begin{aligned}
\left\|q_{i}^{k}\right\|_{i}^{2} & =\left\langle q_{i}^{k}, q_{i}^{k}\right\rangle+\left(q_{i}^{k}, q_{i}^{k}\right) \\
& \geq \frac{\Delta y^{2}(1-\alpha)^{3}}{4 \pi^{2}(1+\alpha)^{3}}\left(q_{i}^{k}, q_{i}^{k}\right)+\left(q_{i}^{k}, q_{i}^{k}\right) \\
& =\left(1+\frac{\Delta y^{2}(1-\alpha)^{3}}{4 \pi^{2}(1+\alpha)^{3}}\right)\left(q_{i}^{k}, q_{i}^{k}\right.
\end{aligned}
$$

We shall write $\gamma=\left(1+\frac{\Delta y^{2}(1-\alpha)^{3}}{4 \pi^{2}(1+\alpha)^{3}}\right)^{1 / 2}>1$ and then we have

$$
\left\|q_{i}^{k}\right\|_{i} \geq \gamma\left(q_{i}^{k}, q_{i}^{k}\right)^{1 / 2}
$$


Now, by substitying the above inequality in (3.6) we get:

$$
\left\|q_{i}^{k}\right\|_{i}^{2} \leq \frac{\alpha \Delta y^{2}}{2(1-\alpha)^{3}}(2 \pi)^{1 / 2} \frac{\left\|q_{i}^{k}\right\|_{i}}{\gamma}+\frac{\left\|q_{i+1}^{k-1}\right\|_{i+1}\left\|q_{i}^{k}\right\|_{i}}{2 \gamma^{2}}+\frac{\left\|q_{i-1}^{k}\right\|_{i-1}\left\|q_{i}^{k}\right\|_{i}}{2 \gamma^{2}}
$$

This inequality holds for all $\mathrm{i}=1, \ldots, \mathrm{N}$, then it holds for the maximum component, so that

$$
\left\|q^{k}\right\|^{2} \leq \frac{\alpha \Delta y^{2}}{2(1-\alpha)^{3}}(2 \pi)^{1 / 2} \frac{\left\|q^{k}\right\|}{\gamma}+\frac{\left\|q^{k-1}\right\| \cdot\left\|q^{k}\right\|}{2 \gamma^{2}}+\frac{\left\|q^{k}\right\|^{2}}{2 \gamma^{2}} .
$$

Then

$$
\left(1-\frac{1}{2 \gamma^{2}}\right)\left\|q^{k}\right\| \leq \frac{\alpha \Delta y^{2}}{2(1-\alpha)^{3}} \frac{(2 \pi)^{1 / 2}}{\gamma}+\frac{\left\|q^{k-1}\right\|}{2 \gamma^{2}}
$$

and

$$
\left\|q^{k}\right\| \leq \frac{\alpha \Delta y^{2}}{2(1-\alpha)^{3}} \frac{\gamma(2 \pi)^{1 / 2}}{2 \gamma^{2}-1}+\frac{\left\|q^{k-1}\right\|}{2 \gamma^{2}-1}
$$

For $\Delta y$ small enough $\left(\frac{\alpha \gamma(2 \pi)^{1 / 2}}{(1-\alpha)^{3}} \Delta y^{2}<1\right)$ we obtain:

$$
\left\|q^{k}\right\| \leq \frac{1}{2 \gamma^{2}-1}+\frac{1}{2 \gamma^{2}-1}\left\|q^{k-1}\right\|
$$

and then

$$
\left\|q^{k}\right\| \leq \frac{1-\beta^{k}}{1-\beta}+\beta^{k}\left\|q^{0}\right\|
$$

where $\beta=\frac{1}{2 \gamma^{2}-1}<1$. Finally we get

$$
\left\|q^{k}\right\| \leq \frac{1}{1-\beta}+\left\|q^{0}\right\|
$$

Proposition 3.2. There exit $q^{*}=\left(q_{1}^{*}, \ldots, q_{N}^{*}\right) \in W$ and $h^{*}=\left(h_{1}^{*}, \ldots, h_{N}^{*}\right) \in\left(L^{\infty}(0,2 \pi)\right)^{N}$ such that

$$
\begin{aligned}
& q^{k} \rightarrow q^{*} \text { weakly in } W \\
& h^{k} \rightarrow h^{*} \text { weak- }^{*} \text { in }\left(L^{\infty}(0,2 \pi)\right)^{N}
\end{aligned}
$$

Proof. Convergence (3.8) is a consequence of proposition 3.1.

For all $k \geq 1$ we have $h^{k}(x) \leq 1+\alpha$ where $\alpha$ is the constant introduced in (1.1). Thus (3.9) holds. 


\section{NumericAl SOLUTION}

We present in this section some numerical results obtained by applying the above algorithm. In this example the gap function is $h(x)=1+0.9 \cos (x)$ and the alimentation pressure is $p_{a}=0.1$. In the figure 3 we present the evolution of the pressure along the algorithm (3.3)(3.4). And in the figure 4 we represent the relative error in the algorithm, that is, at each iteration $k$ we give the error $\frac{\left\|q_{k}-q_{k-1}\right\|}{\left\|q_{k}\right\|}$ being $\|$.$\| the L^{2}(\Omega)$ norm.
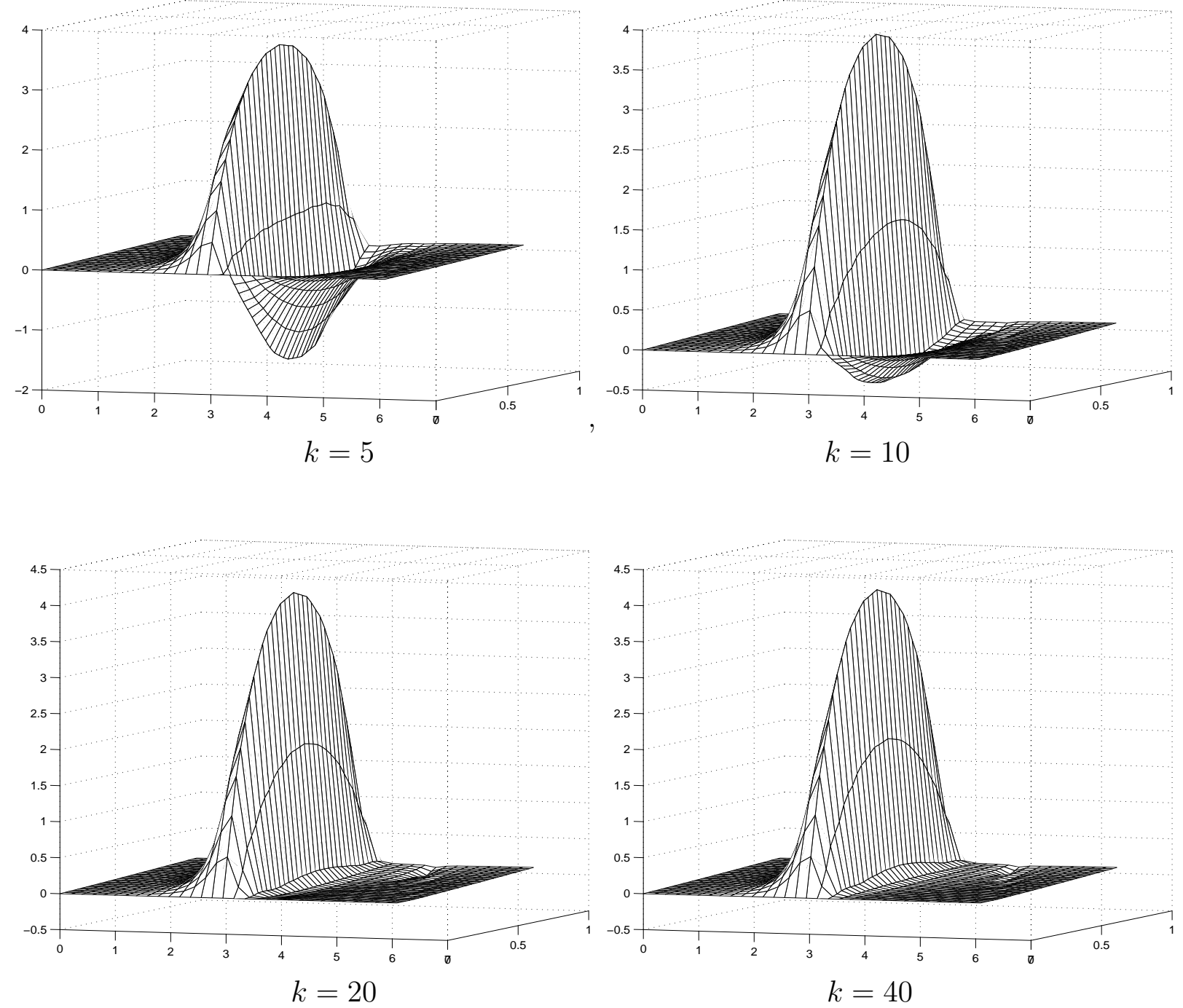

Fig. 3: Evolution of the pressure 


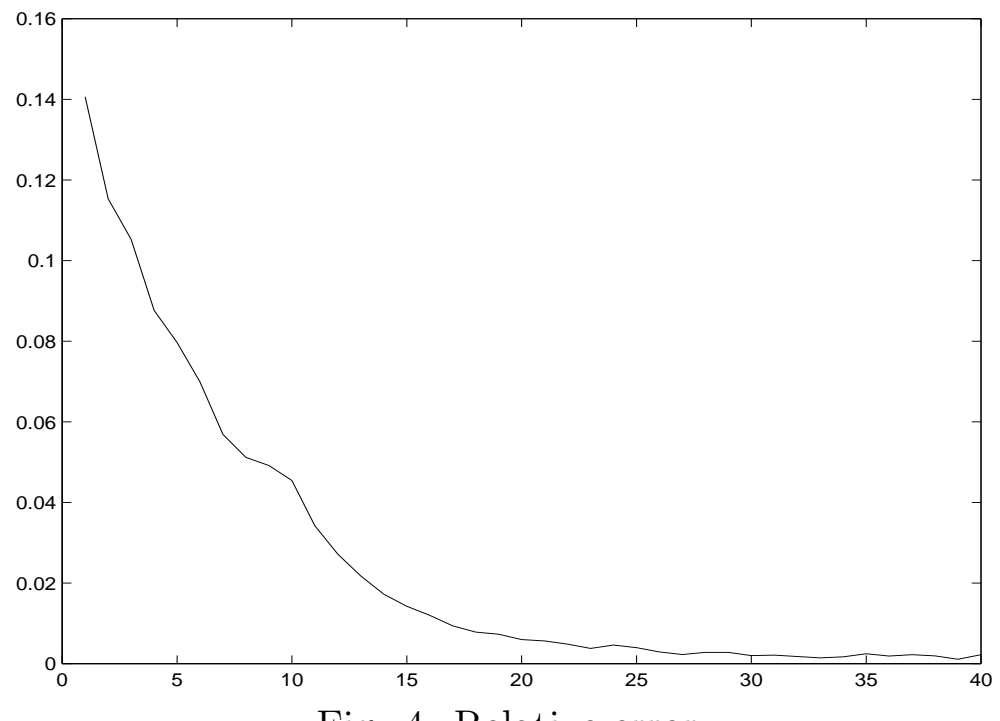

Fig. 4: Relative error

\section{REFERENCES}

[1] S. J. Alvarez Problemas de frontera libre en teoria de lubrificacion Tesis Doctoral, Universidad Complutense de Madrid, 1986

[2] Alvarez S.J. Oujja R. An iterative method for solving a free bondary problem for an infinite journal bearing Applied Mathematics and Computation 122 p.p. 15-26 July (2001)

[3] G. Bayada, M. Chambat Sur quelques modélisations de la zone de cavitation en lubrification hydrodynamique J. of Theor. and Appl. mech., 5(59) 1986

[4] M. El Alaoui Sur un problème À frontière libre en mÉCAnique des films minces Thèse, Universidad de Lyon I, 1986

[5] H. G. ELROD, M. ADAMS, A computer program for Cavitation Proceedings cavitaion and related phenomena in lubrication. Mech. Eng. Publ., London 1975.

[6] R. S. Falk Error estimates for the approximation of a Class of variational inequalities, Math. Comp., 28 (1974), pp. 963-971.

[7] H. Lewy G.Stampacchia On the REgUlarity of the SOlution of a VARIATIONAL INEQUALity Comm. Pure Appl. Math., 22 (1969), pp. 153-188. 